\title{
Diversity of aquatic Angiosperms and Associated Species of Heranj Wetland, Kheda - Gujarat for Sustainability
}

Jaivin Patel, Dr. Bharat Maitreya

Department of Botany, Bioinformatics and climate Change Impacts Management, School of sciences, Gujarat University, Ahmedabad, Gujarat, India

Article Info

Volume 8, Issue 2

Page Number : 214-222

Publication Issue

March-April-2021

\section{Article History}

Accepted : 16 March 2021

Published : 22 March 2021

\section{ABSTRACT}

Wetlands are one of the most productive and fertile ecosystems on earth. They cover about $6.4 \%$ of the land area of the earth (Mitsch and Gosselink, 1986). Wetlands are tremendously valuable pools of biodiversity and genetic resources, but unmaintainable development is intimidating the bio-wealth and even initiating species extinction. Floodplains, mangroves, sea grasses, saltmarshes, arctic wetlands, peat lands, freshwater marshes and forests are very diverse habitats, with different stressors and aquatic angiosperms act as primary creators in aquatic ecosystems and they maintain energy flow in whole bionetwork. Wetland systems are vulnerable to fluctuations in quantity and quality of their water supply, and it is expected that climate change will have a pronounced effect on wetlands through alterations in hydrological regimes with great comprehensive variability. Wetland habitat responses to climate change and the implications for restoration will be realized differently on a regional and mega-watershed level, making it important to recognize that specific restoration and management plans will require examination by habitat. The present study reveals a total of 28 species of Aquatic angiosperms were recorded from the Heranj wetland ( Taluka: Matar District: Kheda, Gujarat ) that include 8 species of submerged, 2 species of free floating, 5 species of rooted with floating leaves and 13 species of emergent Aquatic angiosperms. Najas sp., Hydrilla sp., Typha sp., Potamogeton sp. are dominant species in Freshwater wetland and hence, different management and restoration techniques are needed for its sustainability.

Keywords : Aquatic angiosperms, Heranj pond, Wetland, Ecosystems.

\section{INTRODUCTION}

Wetlands are one of the most productive and fertile ecosystems on earth. They cover about $6.4 \%$ of the land area of the earth (Mitsch and Gosselink, 1986). In india, wetland also cover $1-5 \%$ of the land area(SAC, 2011). Gujarat have both coastal and inland wetland habitat diversity (Stanley, 2004). As per SAC 
(2011) Gujarat has total 23,891 wetlands (Both Coastal and Inland wetlands), of which, 9,708 wetlands are those having an area less than 2.25 ha. Total area of these 23,891 wetlands has been estimated at $34,749.50$ sq. $\mathrm{km}$ which accounts for about $17.56 \%$ of geographical area of the State and $22.7 \%$ of the total wetland area of the country. The area of the Gujart State under coastal wetlands (coral reefs, creeks,inter-tidal flats, sand/beach, coastal marsh mangroves, etc.) are approximately 28,071 sq.km and that under inland wetlands (ponds, rivers, tanks, streams, oxbow lake) has been approximately $6,582 \mathrm{sq} . \mathrm{km}$.

The present study of inland wetland has been carried out for knowing the diversity of the aquatic angiosperms and Associated Species of Heranj wetlands of Gujarat. This is a man-made wetland having an Open Water, emergent hydrophytic vegetation growth, water with Nymphaea/Nelumbo cover, water with decomposing submerged vegetation mat as the predominant habitat component within it.

\section{STUDY AREA}

The study covered one Prioritized wetlands sites from Central Gujarat, Viz., Heranj Wetland. Heranj wetland is located in Matar taluka at $22^{\circ} 40^{\prime} 9.7^{\prime \prime} \mathrm{N}$ latitudes and $72^{\circ} 41^{\prime} 28.5^{\prime \prime} \mathrm{E}$ longitudes. It is situated at the distance of just $27 \mathrm{~km}$ north of Tarapur on Tarapur-Kheda State highway, $50 \mathrm{~km}$ from Ahmedabad and $50 \mathrm{~km}$ from Khambhat. The big lake of heranj is approximately $\mathrm{kms}$. in area with depth varying between $2 \mathrm{ft}$ to $10 \mathrm{ft}$.

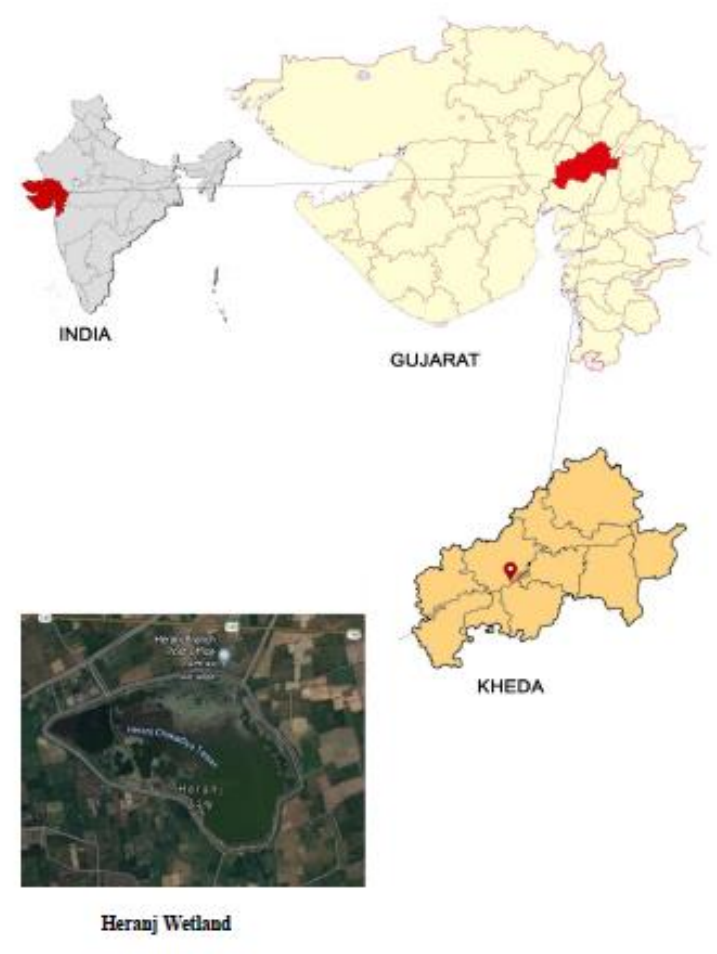

Figure 1: Satellite image of Heranj Wetland (Source: Google Map)

\section{METHODS AND MATERIAL}

The field survey was started with a reconnaissance survey of Heranj Wetland located at Khambhat District of Gujarat state, India. Further, data collection was carried out in each of the season i.e., Summer, Monsoon and Winter. For flora study, a Belt- Transect with stratified random sampling method were carried out. The entire area of wetlands was covered by satisfied sampling and belt transect. The transects were decided and marked in such a way that each transect would represent a type of habitat. The data of flowering plants collected in each season from selected transect-based for habitat stratification. Heranj wetland endowed with six habitat components, i.e. inlet, outlet, open water, emergent hydrophytic vegetation growth, water with Nymphaea/Nelumbo cover, water with decomposing submerged vegetation mat. The plants were identified 
with the help of standard literature and based on micro-morphological plants Characters and flowers.

\section{RESULTS AND DISCUSSION}

\section{Qualitative analysis}

During the present study, a total of 63 species belonging to 32 families and 53 genera of flowering plants have been recorded from the in and around the Heranj wetland. Dicots represented by 40 species belong to 33 genera and 23 families while Monocots represented by 23 species belongs to 20 genera and 9 families. Graph 1 showed habit wise analysis of plants i.e., tree, shrubs, climber, herbs, etc. Furthermore, plant checklist was prepared based on the visual observation in the quadrats as well transects (Table 3 ).

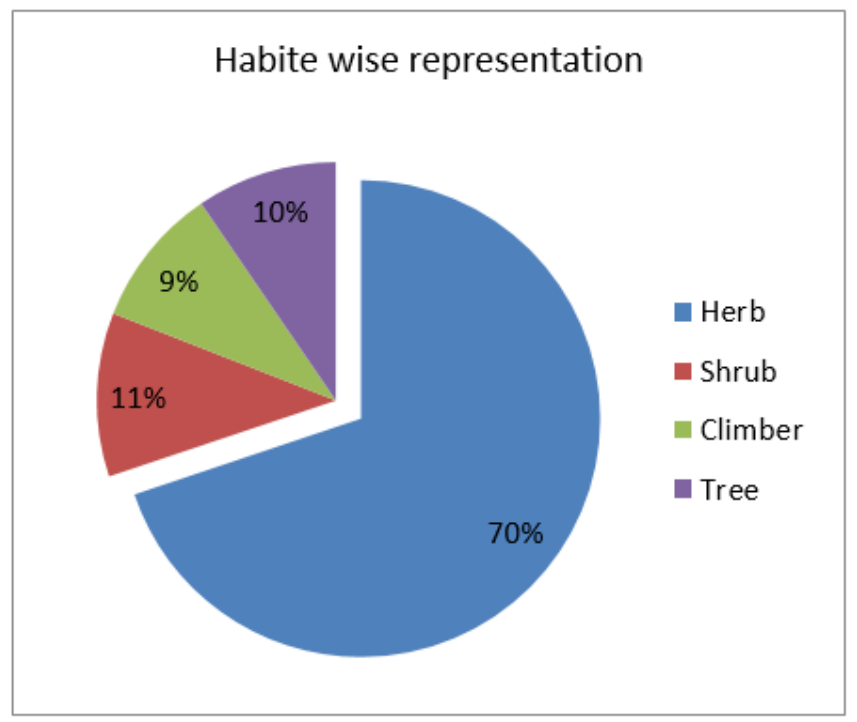

Out of 32 families, 47 genera are represented by single species of each genus. Asteraceae is largest families among the dicot while Poaceae and Cyperaceae are largest among the monocots which are poorly represented. A total of 63 species of flowering plants, 44 species are herbs, 7 species are shrubs, 6 species are climbers and 6 species are trees. This study show that herbaceous plants are dominating in the wetland and its surrounding areas.
Most representative family and genera in Heranj Wetland

Most representative family, genera and species of Heranj are Poaceae ( 7 species), followed by Asteraceae (6 species), Cyperaceae (5 species), Convolvulaceae (5 species), Convolvulaceae (5 species) etc. as given in graph 2 .

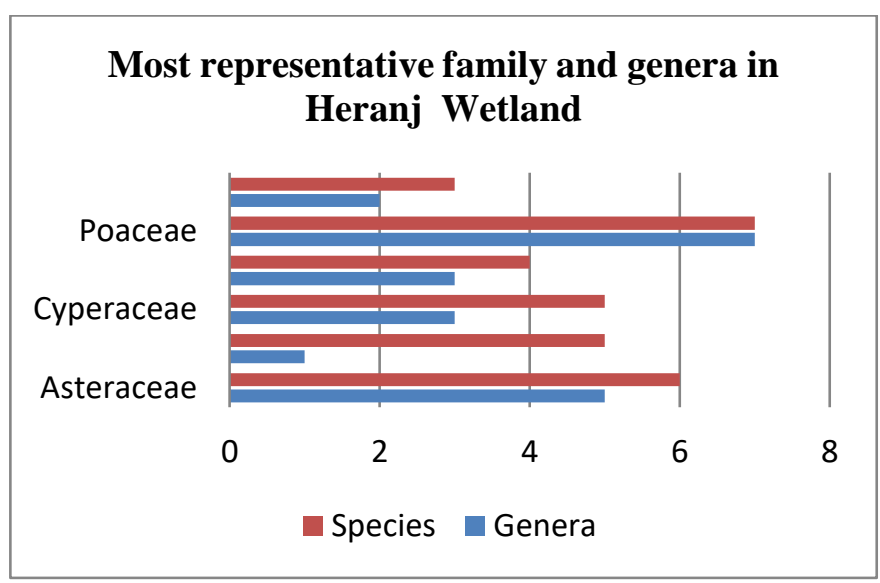

\section{Aquatic Angiosperms}

The Angiosperms are classified into submerged, free floating, rooted floating and emergent vegetation. Total 28 species recorded under this study belonged to 15 families. Majority of the species are recorded from emergent (13 species) vegetation followed by submerged (8 species), Rooted floating (5 species) and Free floating (2 species) Vegetation, etc. (graph 3)

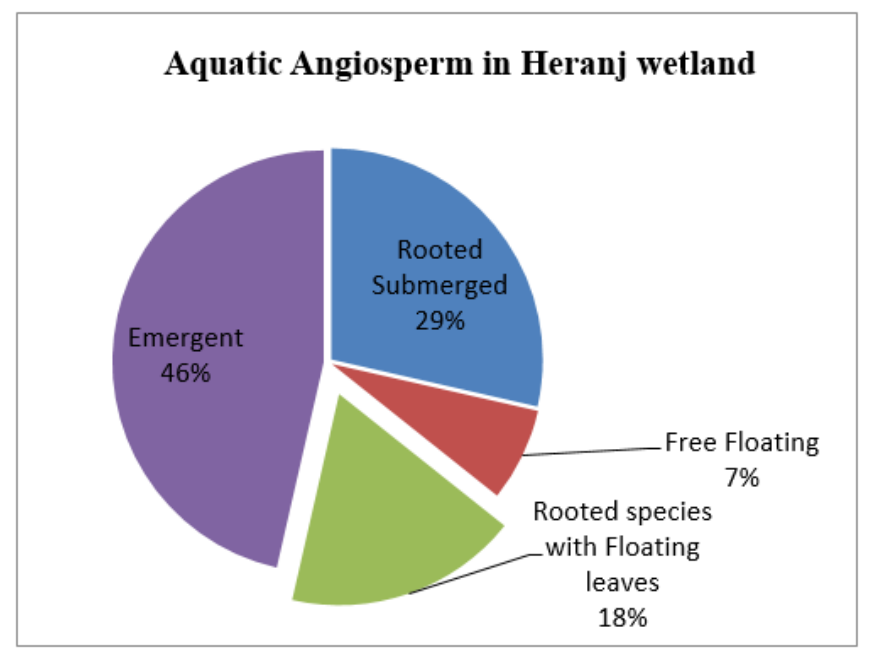


Jaivin Patel, Dr. Bharat Maitreya Int J Sci Res Sci \& Technol. March-April-2021, 8 (2) : 214-222

Table 1: List of Aquatic Angiosperms at Heranj Wetland

\begin{tabular}{|c|c|c|c|c|c|}
\hline Sr. No. & Botanical Name & Family & Status & Habit & $\begin{array}{c}\text { Indicator } \\
\text { status }\end{array}$ \\
\hline 1 & Ammannia baccifera L. & Lythraceae & Emergent & Herb & FAC \\
\hline 2 & Cyperus difformis $\mathrm{L}$. & Cyperaceae & Emergent & Herb & FACW \\
\hline 3 & Cyperus iria $\mathrm{L}$ & Cyperaceae & Emergent & Herb & FACW \\
\hline 4 & Cyperus rotundus $\mathrm{L}$. & Cyperaceae & Emergent & Herb & FACW \\
\hline 5 & Cyperus bulbosus Vahl & Cyperaceae & Emergent & Herb & FACW \\
\hline 6 & $\begin{array}{l}\text { Eichhornia crassipes (Mart.) } \\
\text { Solms }\end{array}$ & Pontederiaceae & $\begin{array}{c}\text { Free } \\
\text { Floating }\end{array}$ & Herb & OBL \\
\hline 7 & Fimbristylis aestivalis Vahl & Cyperaceae & Emergent & Herb & FACW \\
\hline 8 & $\begin{array}{l}\text { Hydrilla verticillata (L.f.) } \\
\text { Royle }\end{array}$ & Hydrocharitaceae & Submerged & Herb & OBL \\
\hline 9 & $\begin{array}{l}\text { Hygrophila auriculata } \\
\text { (Schum.) Heine }\end{array}$ & Acanthaceae & Emergent & Herb & FACW \\
\hline 10 & Ipomoea aquatica Forssk. & Convolvulaceae & $\begin{array}{l}\text { Rooted } \\
\text { Floating }\end{array}$ & Climber & FACW \\
\hline 11 & Ipomoea carnea Jacq. & Convolvulaceae & Emergent & Climber & FACW \\
\hline 12 & $\begin{array}{l}\text { Ipomoea marginata (Desr.) } \\
\text { Verdc. }\end{array}$ & Convolvulaceae & $\begin{array}{l}\text { Rooted } \\
\text { Floating }\end{array}$ & Climber & FACW \\
\hline 13 & Ipomoea triloba L. & Convolvulaceae & $\begin{array}{l}\text { Rooted } \\
\text { Floating }\end{array}$ & Climber & FACW \\
\hline 14 & Lemna minor L. & Lemnaceae & $\begin{array}{c}\text { Free } \\
\text { Floating }\end{array}$ & Herb & OBL \\
\hline 15 & $\begin{array}{l}\text { Limnophyton obtusifolium } \\
\text { (L.) Miq. }\end{array}$ & Alismataceae & Emergent & Herb & FACW \\
\hline 16 & $\begin{array}{l}\text { Ludwigia adscendens (L.) } \\
\text { Hara }\end{array}$ & Onagraceae & Emergent & Herb & FACW \\
\hline 17 & Najas marina L. & Hydrocharitaceae & Submerged & Herb & OBL \\
\hline 18 & Najas minor All. & Hydrocharitaceae & Submerged & Herb & OBL \\
\hline 19 & Nelumbo nucifera Gaertn. & Nelumbonaceae & $\begin{array}{l}\text { Rooted } \\
\text { Floating }\end{array}$ & Herb & OBL \\
\hline 20 & Nymphaea nouchali Burm.f. & Nymphaeaceae & $\begin{array}{l}\text { Rooted } \\
\text { Floating }\end{array}$ & Herb & OBL \\
\hline 21 & $\begin{array}{l}\text { Persicaria glabra (Willd.) } \\
\text { M.Gómez }\end{array}$ & Polygonaceae & Emergent & Herb & FACW \\
\hline 22 & Potamogeton crispus L. & Potamogetonaceae & Submerged & Herb & OBL \\
\hline 23 & Potamogeton nodosus Poir. & Potamogetonaceae & Submerged & Herb & OBL \\
\hline 24 & Scirpus littoralis Schrad. & Cyperaceae & Emergent & Herb & FACW \\
\hline 25 & Stuckenia pectinata (L.) & Potamogetonaceae & Submerged & Herb & OBL \\
\hline
\end{tabular}


Jaivin Patel, Dr. Bharat Maitreya Int J Sci Res Sci \& Technol. March-April-2021, 8 (2) : 214-222

\begin{tabular}{|c|l|c|c|c|c|}
\hline & Börner & & & & \\
\hline 26 & Typha angustifolia L. & Typhaceae & Emergent & Herb & FACW \\
\hline 27 & Utricularia inflexa Forssk. & Lentibulariaceae & Submerged & Herb & OBL \\
\hline 28 & Vallisneria spiralis L. & Hydrocharitaceae & Submerged & Herb & OBL \\
\hline
\end{tabular}

(* Obligate Wetland Plants (OBL), Facultative Wetland Plants (FACW), Facultative Plants(FAC), Facultative Upland Plants (FACU), Obligate Upland Plants (UPL)).

During fieldwork at Heranj wetland 28 species of aquatic macropytes were identified and listed based on the visual observation in the quadrats as well transects by using Cook (1996) and Shah (1978). The given table show list of aquatic macrophytes with their indicator statuses, habit, family, class etc.(Table:1)

\section{Wetland indicator status}

The National List of Plant Species that Occur in Wetlands is a list of wetland plants and their assigned indicator statuses. The five indicator statuses are: Obligate Wetland Plants (OBL), Facultative Wetland Plants (FACW), Facultative Plants (FAC), Facultative Upland Plants (FACU), Obligate Upland Plants (UPL). Based on these indicator statuses total of 63 species of plants were categories and represented. Out of these 31 species belong to Obligate Upland Plants, 15 Facultative Wetland Plants, 12 Obligate Wetland Plants, 5 Facultative Upland Plants and 1naturalized, 1 Facultative Plants. (Graph 4.)

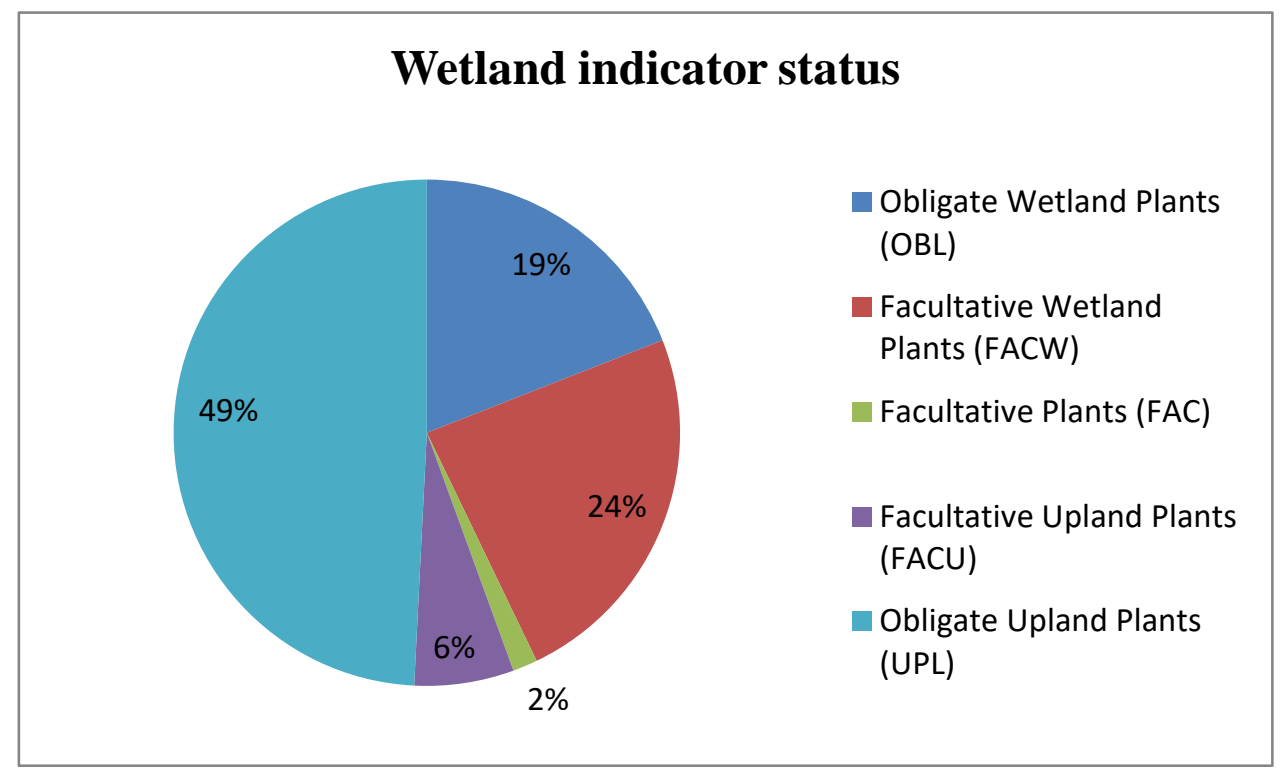


Jaivin Patel, Dr. Bharat Maitreya Int J Sci Res Sci \& Technol. March-April-2021, 8 (2) : 214-222

Table 2. Role of Species involved in wetland Sustainability:

\begin{tabular}{|c|l|l|l|}
\hline $\begin{array}{c}\text { Sr. } \\
\text { No }\end{array}$ & \multicolumn{1}{|c|}{ Species } & \multicolumn{1}{|c|}{ Role } & \multicolumn{1}{c|}{ References } \\
\hline 1 & Hydrilla verticillata & Cleaning of wetland water & $\begin{array}{l}\text { Toth \& } \\
\text { Anderson., 1998 }\end{array}$ \\
\cline { 3 - 5 } & & Helps in Phosphorus removal & Gu., 2006. \\
\hline 2 & Ipomoea aquatica & $\begin{array}{l}\text { Bio-accumulation lead, cadmium and } \\
\text { arsenic }\end{array}$ & (Ghosh, 2010) \\
\hline 3 & $\begin{array}{l}\text { Eichhornia crassipes and } \\
\text { Hydrilla verticillata }\end{array}$ & Phosphorus removal efficiency & Shardendu et al., \\
\hline 5 & Scirpus Sp. & $\begin{array}{l}\text { Sequential nitrogen detoxification, } \\
\text { removal of BOD and TSS of }\end{array}$ & Brix,1997 \\
\hline 6 & Typha & Removing ammonia & Brix,1997 \\
\hline 7 & $\begin{array}{l}\text { Eleocharis spp, Nymphaea } \\
\text { odorata }\end{array}$ & Increases nitrogen content & Brix,1997 \\
\hline
\end{tabular}

Table 3 : Check list of flowering plants of Heranj Wetland

\begin{tabular}{|c|l|c|c|c|}
\hline $\begin{array}{c}\text { Sr. } \\
\text { No. }\end{array}$ & Botanical Name & Local name & Family & Habit \\
\hline 1 & Abutilon indicum (L.) Sw. & $\begin{array}{c}\text { Mudra, Petari, } \\
\text { Karandi }\end{array}$ & Malvaceae & Shrub \\
\hline 2 & Achyranthes aspera L. & Andhedi & Amaranthaceae & Herb \\
\hline 3 & $\begin{array}{l}\text { Alternanthera sessilis (L.) R.Br. ex } \\
\text { DC. }\end{array}$ & - & Amaranthaceae & Herb \\
\hline 4 & Ammannia baccifera L. & Jal agiyo & Lythraceae & Herb \\
\hline 5 & Azadirachta indica (L.) Juss. & Neem, Limdo & Meliaceae & Tree \\
\hline 6 & Blumea lacera (Burm.f.) DC. & - & Asteraceae & Herb \\
\hline 7 & Blumea mollis (D. Don) Merr. & - & Asteraceae & Herb \\
\hline 8 & Calotropis procera (Aiton) Dryand. & Akado & Asclepiadaceae & Shrub \\
\hline 9 & Chloris barbata Sw. & - & Poaceae & Herb \\
\hline 10 & Cocculus hirsutus (L.) W.Theob. & Vevdi & Menispermaceae & Climber \\
\hline 11 & Coix lacryma-jobi L. & - & Poaceae & Herb \\
\hline 12 & Commelina benghalensis L & - & Commelinaceae & Herb \\
\hline 13 & Cyanthillium cinereum (L.) H.Rob. & - & Asteraceae & Herb \\
\hline
\end{tabular}


Jaivin Patel, Dr. Bharat Maitreya Int J Sci Res Sci \& Technol. March-April-2021, 8 (2) : 214-222

\begin{tabular}{|c|c|c|c|c|}
\hline 14 & Cynodon dactylon (L.) Pers. & Darbh & Poaceae & Herb \\
\hline 15 & Cyperus difformis $\mathrm{L}$. & - & Cyperaceae & Herb \\
\hline 16 & Cyperus iria L & - & Cyperaceae & Herb \\
\hline 17 & Cyperus rotundus $\mathrm{L}$. & Dilo & Cyperaceae & Herb \\
\hline 18 & $\begin{array}{l}\text { Dichanthium annulatum (Forssk.) } \\
\text { Stapf }\end{array}$ & Darbha & Poaceae & Herb \\
\hline 19 & $\begin{array}{l}\text { Digitaria abludens (Roem. \& Schult.) } \\
\text { Veldkamp }\end{array}$ & Bondya & Poaceae & Herb \\
\hline 20 & Eclipta prostrata (L.) L. & - & Asteraceae & Herb \\
\hline 21 & Eichhornia crassipes (Mart.) Solms & - & Pontederiaceae & Herb \\
\hline 22 & Eragrostis tenella (L.) P. Beauv. & - & Poaceae & Herb \\
\hline 23 & Euphorbia hirta L. & - & Euphorbiaceae & Herb \\
\hline 24 & Ficus virens Dryand. ex Ait. & - & Moraceae & Tree \\
\hline 25 & Fimbristylis aestivalis Vahl & - & Cyperaceae & Herb \\
\hline 26 & Glinus lotoides L. & - & Molluginaceae & Herb \\
\hline 27 & Grangea maderaspatana (L.) Poir. & - & Asteraceae & Herb \\
\hline 28 & Heliotropium supinum L. & $\begin{array}{c}\text { Prostrate } \\
\text { Heliotrope }\end{array}$ & $\underline{\text { Boraginaceae }}$ & Herb \\
\hline 29 & Hydrilla verticillata (L.f.) Royle & - & Hydrocharitaceae & Herb \\
\hline 30 & $\begin{array}{l}\text { Hygrophila auriculata (Schum.) } \\
\text { Heine }\end{array}$ & - & Acanthaceae & Herb \\
\hline 31 & Ipomoea aquatica Forssk. & Vel & Convolvulaceae & Climber \\
\hline 32 & Ipomoea carnea Jacq. & $\begin{array}{l}\text { Bush Morning } \\
\text { Glory }\end{array}$ & Convolvulaceae & Climber \\
\hline 33 & Ipomoea marginata (Desr.) Verdc. & - & Convolvulaceae & Climber \\
\hline 34 & Ipomoea pes-tigridis L. & - & Convolvulaceae & Climber \\
\hline 35 & Ipomoea triloba L. & - & Convolvulaceae & Climber \\
\hline 36 & Lantana camara L. & - & Verbenaceae & Shrub \\
\hline 37 & $\begin{array}{l}\text { Launaea procumbens (Roxb.) } \\
\text { Ramayya \& Rajagopal }\end{array}$ & Gadjepi & Asteraceae & Herb \\
\hline 38 & Lawsonia inermis $\mathrm{L}$. & Mahendi & Lythraceae & Shrub \\
\hline 39 & Lemna minor L. & - & Lemnaceae & Herb \\
\hline 40 & Limnophyton obtusifolium (L.) Miq. & - & Alismataceae & Herb \\
\hline 41 & Ludwigia adscendens (L.) Hara & - & Onagraceae & Herb \\
\hline 42 & Mangifera indica L. & Ambo & Anacardiaceae & Tree \\
\hline 43 & Najas marina L. & - & Hydrocharitaceae & Herb \\
\hline 44 & Nelumbo nucifera Gaertn. & Kamal & Nelumbonaceae & Herb \\
\hline 45 & Nymphaea nouchali Burm.f. & Poynu & Nymphaeaceae & Herb \\
\hline 46 & Polygonum plebeium R.Br. & - & Polygonaceae & Herb \\
\hline 47 & Potamogeton crispus L. & - & Potamogetonaceae & Herb \\
\hline 48 & Potamogeton nodosus Poir. & - & Potamogetonaceae & Herb \\
\hline
\end{tabular}


Jaivin Patel, Dr. Bharat Maitreya Int J Sci Res Sci \& Technol. March-April-2021, 8 (2) : 214-222

\begin{tabular}{|c|l|c|c|c|}
\hline 49 & Prosopis cineraria (L.) Druce & Khijdo & Leguminosae & Tree \\
\hline 50 & Prosopis juliflora (Sw.) DC. & Gando Baval & Leguminosae & Shrub \\
\hline 51 & Rungia repens (L.) Nees & - & Acanthaceae & Herb \\
\hline 52 & Salvadora persica L. & Piludi & Salvadoraceae & Shrub \\
\hline 53 & Scirpus littoralis Schrad. & - & Cyperaceae & Herb \\
\hline 54 & Senna auriculata (L.) Roxb. & Tarvad, Awal & Leguminosae & Tree \\
\hline 55 & Sida cordifolia L & - & Malvaceae & Herb \\
\hline 56 & Solanum surattense Burm. f. & Boyringni & Solanaceae & Herb \\
\hline 57 & $\begin{array}{l}\text { Sporobolus coromandelianus } \text { (Retz.) } \\
\text { Kunth }\end{array}$ & - & Poaceae & Herb \\
\hline 58 & Stuckenia pectinata (L.) Börner & - & Potamogetonaceae & Herb \\
\hline 59 & Typha domingensis Pers. & Gha Bajariu & Typhaceae & Herb \\
\hline 60 & $\begin{array}{l}\text { Vachellia nilotica (L.) P.J.H. Hurter \& } \\
\text { Mabb. }\end{array}$ & Baval & Leguminosae & Herb \\
\hline 61 & Vallisneria spiralis L. & - & Hydrocharitaceae & Herb \\
\hline 62 & Ziziphus jujuba Mill. & Bor & Rhamnaceae & Tree \\
\hline 63 & $\begin{array}{l}\text { Ziziphus nummularia (Burm.f.) } \\
\text { Wight \& Arn }\end{array}$ & Rhamnaceae & Shrub \\
\hline
\end{tabular}

\section{v. CONCLUSION}

Anthropogenic activities have impacted on wetland ecosystem services across the world. Restoration of wetlands are needed. Present study show the species composition of aquatic angiosperm are play vital role for wetland sustainability as well as it can be consider throughout the wetland restoration activity. Study will also helpful to recreate wetland diversity precisely to Kheda District which have maximum number of wetland in Gujarat state. Furthermore, study can be also helpful for understanding the complexity of wetland ecosystem.

\section{VI.ACKNOWLEDGMENT}

The authors are thankful to SHODH-Scheme Of Developing High quality research, Education Department, Gujarat State for facilitating Scholarship and also Gratitude for Gujarat Ecological Education and Research (GEER) Foundation, Gandhinagar for facilitating various training and workshop support. Gratitude are also due to the Gujarat Forest
Department authorities managing Heranj wetlands. I particularly express my deep and sincere sense of gratitude to Mr. Rupesh Maurya who took special interest in my study and gave me valuable inputs during my entire work. I always remain indebted to Dr. Archna A. Mankad, Professor \& Head, Department of Botany, University School of Sciences, Gujarat University, Ahmedabad. Her constructive criticism, continuous support and much needed motivation helped me to improve my work thought the tenure of my research.

\section{REFERENCES}

[1]. Brix, H. (1997). Do macrophytes play a role in constructed treatment wetlands?. Water science and technology, 35(5), 11-17.

[2]. Cooke, G. D., Welch, E. B., Peterson, S., \& Nichols, S. A. (2016). Restoration and management of lakes and reservoirs. CRC press. 
[3]. Ghosh, S. (2010). Wetland macrophytes as toxic metal accumulators. International Journal of Environmental Sciences, 1(4), 523-528.

[4]. Gu, B. (2006). Environmental conditions and phosphorus removal in Florida lakes and wetlands inhabited by Hydrilla verticillata (Royle): implications for invasive species management. Biology

[5]. Mitsch WJ, Gosselink G. Wetlands. New York: Van Nostrand Reinhold; 1986.

[6]. SAC. National Wetland Atlas. Ahmedabad, India: SAC/EPSA/ABHG/N WIAATLAS/34/2011, Space Applications Centre (ISRO); 2011.

[7]. Shah, G. L. (1978). Flora of Gujarat state.

[8]. Shardendu, S., Sayantan, D., Sharma, D., \& Irfan, S. (2012). Luxury uptake and removal of phosphorus from water column by representative aquatic plants and its implication for wetland management. ISRN Soil Science, 2012.

[9]. Stanley, O. D. (2004). Wetland ecosystems and coastal habitat diversity in Gujarat, India. Journal of coastal development, 7(2), 49-64.

[10]. Toth, L. A., \& Anderson, D. H. (1998, March). Developing expectations for ecosystem restoration. In Transactions of the North American Wildlife and Natural Resources Conference(Vol. 63, pp. 122-134). Wildlife Management Institute.

[11]. Zweig, C. L., \& Kitchens, W. M. (2008). Effects of landscape gradients on wetland vegetation communities: information for large-scale restoration. Wetlands, 28(4), 1086-1096.

\section{Cite this article as :}

Jaivin Patel, Dr. Bharat Maitreya, "Diversity of aquatic Angiosperms and Associated Species of Heranj Wetland, Kheda - Gujarat for Sustainability ", International Journal of Scientific Research in Science and Technology (IJSRST), Online ISSN : 2395-602X, Print ISSN : 2395-6011, Volume 8 Issue 2, pp. 214-222, March-April 2021. Available at doi : https://doi.org/10.32628/IJSRST218219 Journal URL : https://ijsrst.com/IJSRST218219

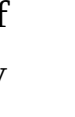

